\title{
Comparações entre os valores genéticos para características de crescimento de bovinos da raça Nelore preditos com modelos de dimensão finita ou infinita ${ }^{1}$
}

\author{
Lucia Galvão de Albuquerque ${ }^{2}$, Lenira El Faro ${ }^{3}$
}

\author{
1 Suporte financeiro da Fapesp e do CNPq. \\ 2 DZ - MGA - FCAV - Unesp - Jaboticabal-SP. Pesquisador do CNPq. \\ 3 PRDTA de Ribeirão Preto - APTA/SAA/SP.
}

\begin{abstract}
RESUMO - Foram utilizados 20.065 pesos de 3.016 animais da raça Nelore com o objetivo de comparar os valores genéticos para características de crescimento, preditos pelo modelo de regressão aleatória (RA) sobre polinômios de Legendre da idade (PL) e pelo modelo de regressão aleatória sobre polinômios de Legendre associada a uma estrutura de correlações paramétricas para modelar as correlações entre diferentes medidas do mesmo animal (FC). Análises bicaracterísticas (BC) para as idades-padrão também foram realizadas. Todos os modelos incluíram o efeito de grupo contemporâneo como fixo e a idade da mãe (efeitos linear e quadrático) como co-variável. Para as análises de RA, as tendências médias foram modeladas por uma regressão cúbica sobre polinômios ortogonais da idade do animal. As ordens dos polinômios foram 4, 4, 6 e 3 para os efeitos genéticos direto e materno, de ambiente permanente direto e materno, respectivamente. Alternativamente, correlações entre diferentes medidas do mesmo animal foram modeladas por uma função paramétrica não-estacionária. Para os resíduos, considerou-se uma função de variâncias ou classes de resíduos. Comparações dos valores genéticos preditos para as idadespadrão; por análises BC e por PL e FC, comprovaram que ocorreram diferenças importantes na classificação dos animais quando se passou de um modelo de dimensão finita para um de dimensão infinita. As correlações de posto entre os valores genéticos preditos usando PL e os preditos usando FC foram próximas da unidade.
\end{abstract}

Palavras-chave: análises bicaracterísticas, avaliação genética, função de correlação paramétrica, polinômios de Legendre

\section{Comparison of breeding values for growth traits in Nellore cattle predicted by finite or infinite dimension model}

\begin{abstract}
Data consisting of 20,065 weights of 3,016 Nellore animals recorded from birth to 630 days of age were used to compare random regression on Legendre polynomials of age (LP) and on Legendre polynomials associated to a parametric correlation structure (PC) models for breeding value prediction of growth traits. Results from Standard bivariate analyses (SBA) using weights at standard ages were also compared to those from random regression models. Dependent variables in random regression analyses were Legendre polynomials of age at recording and the models included contemporary groups as fixed effects, and age of dam with linear and quadratic components as covariates. Mean trends were modeled through a cubic regression on orthogonal polynomials of age and genetic direct and maternal and direct and maternal permanent environmental effects were modeled by polynomials of orders of 4, 4, 6 and 3 respectively. Measurement errors were modeled through a variance function or by considering seven classes of residuals. Also, a non stationary parametric correlation function was used to model correlations between different weights on the same animal. The rank of animals based on breeding values predicted by SBA differed from those predicted by infinite dimension models. Rank correlations between breeding values predicted using LP for animal permanent environmental effects and those predicted using PC were close to unity.
\end{abstract}

Key Words: genetic evaluation, Legendre polynomials, parametric correlation function, standard bivariate analysis

\section{Introdução}

A avaliação genética e posterior seleção dos animais depende, entre outros fatores, da disponibilidade de estimativas de parâmetros genéticos para as características de interesse. Existe ampla literatura relatando valores estimados de herdabilidade, repetibilidade e correlações genéticas para diversas características em inúmeras raças e espécies animais. Em gado de corte, normalmente, para características de crescimento, os valores genéticos são preditos e os componentes de variância são estimados considerando o peso às idades-padrão como, por exemplo, nascimento, 
desmama, ano e final; ou os ganhos de peso entre estas idades utilizando-se análises uni ou multicaracterísticas.

Estimativas de co-variâncias para características de crescimento são abundantes, principalmente para Bos taurus, em regiões temperadas (Mohiuddin, 1993; Koots et al., 1994a,b). No Brasil, Mercadante et al. (1995) e Lôbo et al. (2000), em trabalhos de revisão, apresentaram estimativas médias de herdabilidade para pesos em diferentes idades nos trópicos, para as raças Zebu e todas as raças, respectivamente, semelhantes às descritas por Koots et al. (1994a).

Alternativamente, funções de co-variância e modelos de regressão aleatória (MRA) têm sido usados para modelar características medidas repetidamente na vida dos animais, denominadas características repetidas ou dados longitudinais (Kirkpatrick et al., 1990, 1994; Schaeffer \& Dekkers, 1994). Uma função de co-variâncias é o equivalente de "dimensão infinita" a uma matriz de co-variâncias em uma análise multicaracterística (Kirkpatrick \& Heckman, 1989). Os coeficientes das funções de co-variâncias podem ser estimados diretamente dos modelos de regressão aleatória por máxima verossimilhança restrita (Meyer \& Hill, 1997; Meyer, 1998a).

Em contraste aos modelos multicaracterísticos, que fornecem predições para determinados pontos ou idades, os modelos de regressão aleatória permitem a predição de valores genéticos para a curva de crescimento como um todo para qualquer idade desejada e para funções da curva de crescimento. Esses mesmos modelos têm sido utilizados para modelar grande variedade de características em diversas espécies, como suínos, bovinos de leite e de corte, com destaque principalmente para a modelagem de dados de produção de leite no dia do controle em bovinos de leite (Jamrozik et al., 1997; Van der Werf et al., 1998). Em gado de corte, ainda são relativamente poucos os trabalhos empregando regressões aleatórias para características de crescimento. Na maioria, foram utilizados pesos em idade adulta ou pós-desmama (Meyer, 1998a, 1998c, 1999, 2000). No Brasil, funções de co-variância foram estimadas para pesos na fase de crescimento para bovinos das raças Nelore (Albuquerque \& Meyer, 2001a; Nobre et al., 2003), Tabapuã (Sakaguti et al., 2003; Dias et al., 2006) e Guzerá (Scarpel, 2004; Silva, 2004).

Nos modelos de regressão aleatória mais utilizados, têm-se empregado regressões sobre polinômios ortogonais de Legendre para modelar as trajetórias fixas e aleatórias (Olori et al., 1999; Meyer, 1998a, 1998c, 1999, 2000; LópezRomero \& Carabanõ, 2003; Strabel et al., 2005). A utilização desses modelos em lugar de análises multicaracterísticas com 4 a 5 pesos nas idades-padrão aumenta o número de parâmetros a serem estimados e os requerimentos computacionais. Uma alternativa é a modelagem das covariâncias de acordo com os efeitos de ambiente permanente de animal, por meio de funções de correlação paramétricas associadas a funções de variâncias, como proposto por Foulley et al. (2000).

Objetivou-se comparar os valores genéticos para o efeito direto preditos empregando-se modelos: bicaracterísticos (BC) de forma padrão; de regressão aleatória sobre polinômios de Legendre da idade (PL); e de regressão aleatória sobre polinômios de Legendre associada a uma estrutura de correlações paramétricas; para modelar as correlações entre diferentes medidas do mesmo animal (FC).

\section{Material e Métodos}

Os dados utilizados neste trabalho foram fornecidos pela Associação Brasileira de Criadores de Zebu (ABCZ). Do conjunto original de pesos de animais da raça Nelore, foram mantidos dados de animais criados a pasto, sem suplementação alimentar. Os animais não receberam tratamento veterinário, nasceram após 1981, eram filhos de vacas com mais de 2 anos e menos de 21 anos de idade; tinham 15 a $50 \mathrm{~kg}$ de peso ao nascer, peso médio diário na média de todos os animais de mesma idade \pm três desviospadrão, tinham no mínimo três pesos válidos (isto é, que atendiam a essas condições) e pertenciam a grupos contemporâneos com mínimo de quatro animais. Quando um dos pesos do animal não atendeu a essas condições, todos os pesos daquele animal foram descartados. Os pesos medidos após 630 dias de idade foram desconsiderados. A definição de grupo contemporâneo incluiu: rebanho, ano e mês de nascimento, sexo, categoria (mamando ou desmamado), ano e mês da pesagem.

Após edição, o conjunto continha 20.065 pesos de 3016 animais, filhos de 87 touros distribuídos em 523 grupos contemporâneos. Todos os animais tinham peso ao nascer com pesagens realizadas a cada 90 dias de idade. Um arquivo de pedigree foi criado considerando até três gerações (Tabela 1).

Tanto as médias como os desvios-padrão aumentaram quase linearmente com a idade do animal (Figura 1) e tiveram decréscimo na velocidade de crescimento após um ano de idade.

A predição dos valores genéticos dos animais foi realizada com os seguintes modelos: (1) bicaracterísticos, utilizando o peso ao nascer como âncora (BC); (2) de regressão aleatória (MRA), sobre polinômios de Legendre 
Tabela 1 - Resumo da estrutura dos dados

\begin{tabular}{lr}
\hline Informação & Número \\
\hline Dados & 20.065 \\
Animais com dados & 3.016 \\
Com três dados & 456 \\
Com quatro dados & 158 \\
Com cinco dados & 5 \\
Com seis dados & 79 \\
Com sete dados & 978 \\
Com oito dados & 1.340 \\
Touros & 87 \\
Vacas & 1.903 \\
Animais nas análises & 5.751 \\
Grupos de contemporâneos & 523 \\
\hline
\end{tabular}

da idade (PL) com ordens de 4, 4, 6 e 3 , respectivamente, para os efeitos genéticos direto $(\mathrm{A})$ e materno $(\mathrm{M})$, ambiente permanente direto $(\mathrm{Q})$ e materno $(\mathrm{C})$ e uma função de variâncias cúbica para o resíduo; (3) de regressão aleatória, sobre polinômios de Legendre da idade com ordens iguais às do modelo (2) para $\mathrm{A}, \mathrm{Me} \mathrm{C}$ e uma função de correlação paramétrica não-estacionária (função de antedependência estruturada) aliada a uma função de variâncias cúbica para o ambiente permanente de animal e classes para o resíduo (FC). As ordens dos polinômios e a função de correlação paramétrica mais adequadas foram obtidas por Albuquerque \& Meyer (2001a) e Albuquerque \& Meyer (2005) para esse mesmo conjunto de dados.

Para que as análises bicaracterísticas pudessem ser comparadas às de regressão aleatória, os dados foram extraídos do mesmo conjunto de dados (Albuquerque \& Meyer, 2001b). Assim, foram utilizados 3.016 dados de pesos ao nascer (PN) e 2.450,2.399 e 2.192 de peso aos 205 (P205), 365 (P365) e 550 (P550) dias de idade, respectivamente, previamente ajustados para idade do bezerro. Foi analisado também o peso aos 120 dias de idade considerando uma pesagem para cada animal, tomada entre 75 e 165 dias de idade.

Os componentes de variância foram estimados pelo método de máxima verossimilhança restrita (Patterson \& Thompson, 1971) não-derivativo (Smith \& Graser, 1986; Graser et al., 1987) empregando modelos animais bicaracterísticos e o algoritmo desenvolvido por Boldman et al. (1993). O modelo geral utilizado pode ser representado na forma matricial como:

$\mathrm{y}=\mathrm{X} \beta+\mathrm{Z}_{1} \mathrm{a}+\mathrm{Z}_{2} \mathrm{~m}+\mathrm{Wc}+\mathrm{e}$

em que: $y=$ vetor dos pesos observados nas diferentes idades; $\beta$ = vetor de efeitos fixos; e $m=$ vetores de efeitos genéticos direto e materno, respectivamente; $\mathrm{c}=$ vetor de efeitos de ambiente permanente; $\mathrm{e}=$ vetor de resíduos; $\mathrm{X}$, $\mathrm{Z}_{1}, \mathrm{Z}_{2}$ e $\mathrm{W}=$ matrizes de incidências para $\mathrm{b}, \mathrm{a}, \mathrm{m}$ e c, respectivamente.

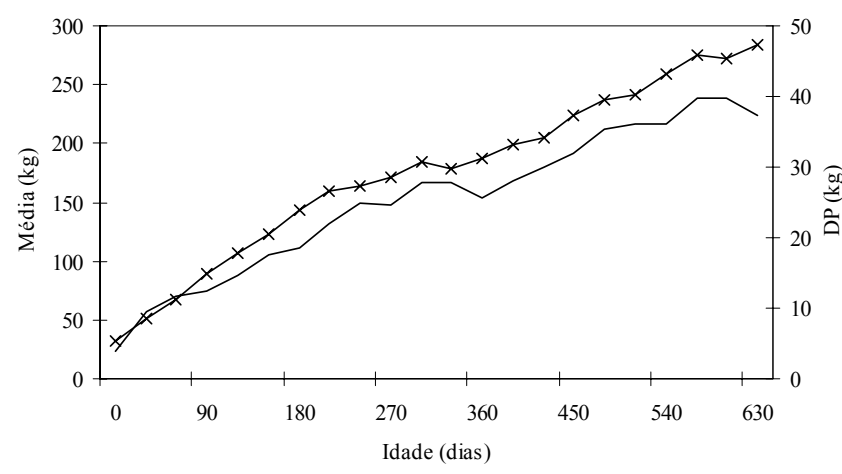

Figura 1 - Médias de peso ( $x$ ) e desvio-padrão (linha) de acordo com a idade do animal.

Assumindo $\mathrm{E}[\mathrm{y}]=\mathrm{X} \beta, \mathrm{E}[\mathrm{a}]=0, \mathrm{E}[\mathrm{m}]=0, \mathrm{E}[\mathrm{c}]=0 \mathrm{e} E[\mathrm{e}]=0, \mathrm{a}$ matriz de co-variâncias é determinada por:

$\left[\begin{array}{c}a_{1} \\ a_{2} \\ m_{1} \\ m_{2} \\ c_{1} \\ c_{2} \\ e_{1} \\ e_{2}\end{array}\right]=\left[\begin{array}{cccccccc}A \sigma_{a_{1}}^{2} & A \sigma_{a_{1} a_{2}} & 0 & 0 & 0 & 0 & 0 & 0 \\ A \sigma_{a_{1} a_{2}} & A \sigma_{a_{2}}^{2} & 0 & 0 & 0 & 0 & 0 & 0 \\ 0 & 0 & A \sigma_{m_{1}}^{2} & A \sigma_{m_{1} m_{2}} & 0 & 0 & 0 & 0 \\ 0 & 0 & A \sigma_{m_{1} m_{2}} & A \sigma_{m_{2}}^{2} & 0 & 0 & 0 & 0 \\ 0 & 0 & 0 & 0 & I_{N_{m}} \sigma_{c_{1}}^{2} & I_{N_{m}} \sigma_{c_{1} c_{2}} & 0 & 0 \\ 0 & 0 & 0 & 0 & I_{N_{m}} \sigma_{c_{1} c_{2}} & I_{N_{m}} \sigma_{c_{2}}^{2} & 0 & 0 \\ 0 & 0 & 0 & 0 & 0 & 0 & I_{N} \sigma_{e_{1}}^{2} & I_{N} \sigma_{e_{1} e_{2}} \\ 0 & 0 & 0 & 0 & 0 & 0 & I_{N} \sigma_{e_{1} e_{2}} & I_{N} \sigma_{e_{2}}^{2}\end{array}\right]$

em que: $\mathrm{Nm}=$ número de mães, $\mathrm{N}$ = número de dados, $\mathrm{A}=$ matriz de parentesco entre os animais; e I = uma matriz identidade. As co-variâncias entre os efeitos genéticos direto e materno; entre os efeitos genéticos e ambientais; e entre os efeitos de ambiente de animais diferentes foram assumidas como zero.

Os modelos incluíram como efeitos fixos o grupo de contemporâneos e a co-variável idade da mãe ao parto (efeitos linear e quadrático). Para P120, os efeitos linear e quadrático da idade do bezerro também foram incluídos.

Em estudos preliminares, verificou-se por meio do teste de razão de verossimilhança que a inclusão do efeito genético materno no modelo não aumentou significativamente a função de verossimilhança para o PN e para os pesos pósdesmama; assim, esse efeito não foi considerado na análise destas características. Entretanto, os resultados desse teste indicaram a necessidade de inclusão do efeito de ambiente permanente materno nos modelos para estas características.

As co-variâncias entre os coeficientes de regressão aleatória foram estimadas por REML usando o programa DXMRR (Meyer, 1998b). Os efeitos fixos e as co-variáveis foram os mesmos utilizados para as análises bicaracterísticas, acrescentando-se uma regressão cúbica sobre polinômios ortogonais de idade para modelar as tendências da média populacional. Polinômios de Legendre de idade foram 
utilizados como variáveis independentes. Os modelos de regressão aleatória foram descritos por Albuquerque \& Meyer (2001a, 2005).

Para as comparações dos valores genéticos e o cálculo da porcentagem de animais coincidentes, foram considerados os números totais de animais (5751) e de touros (260) na matriz de parentesco. Foram realizadas correlações de posto (Spearman) entre os valores genéticos dos animais para PN, P120, P205, P365 e P550, obtidos pelos três modelos: 1 - análises bicaracterísticas padrão, utilizando o peso ao nascer como âncora (BC); 2 - modelo de regressão aleatória sobre polinômios de Legendre da idade, com ordens iguais a 4, 4, 6 e 3, para os efeitos genético direto $\left(\mathrm{k}_{\mathrm{a}}\right)$, genético materno $\left(\mathrm{k}_{\mathrm{m}}\right)$, ambiente permanente de animal $\left(\mathrm{k}_{\mathrm{c}}\right)$ e materno $\left(\mathrm{k}_{\mathrm{q}}\right)$, respectivamente, e uma FV cúbica para modelar as variâncias residuais (PL); e 3 - modelo de regressão alea-

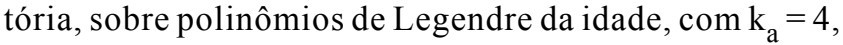
$\mathrm{k}_{\mathrm{m}}=4, \mathrm{k}_{\mathrm{q}}=3$, uma função de correlação paramétrica nãoestacionária (ADE) aliada a uma função de variâncias cúbica para modelar as correlações e variâncias de ambiente permanente de animal e sete classes para o resíduo (FP). $\mathrm{O}$ peso ao nascer foi empregado como âncora nas análises bicaracterísticas, uma vez que foi a única característica medida em todos os animais e considerada nas análises de regressão aleatória. Desse modo, a influência de possíveis imprecisões desta medida estiveram presentes em todas as análises.

Foram também calculados os números de animais machos coincidentes, isto é, que seriam selecionados pelos PL ou FP, quando 10 ou $1 \%$ dos machos fossem escolhidos com base nas análises bicaracterísticas padrão ou quantos seriam selecionados por BC e PL usando-se FP. Para os touros, foram consideradas as porcentagens de 10 e $4 \%$.

\section{Resultados e Discussão}

Os pesos médios, de acordo com as classes de idade (Figura 1), aumentaram quase linearmente com a idade e tiveram decréscimo na taxa de crescimento após a desmama. Os desvios-padrão mostraram a mesma tendência que as médias: aumentaram com a idade e variaram de 2,31 a 45,98 $\mathrm{kg}$. Os coeficientes de variação aumentaram até os animais atingirem 30 a 60 dias de idade e então decresceram levemente com a idade, indicando forte associação entre as médias e os desvios-padrão.

Com as análises bicaracterísticas, as variâncias de ambiente não são decompostas em permanente e temporária, assim, para que as estimativas obtidas com os três modelos fossem comparáveis, estas duas variâncias obtidas com os modelos de regressão aleatória foram somadas (Figura 2).
De modo geral, as estimativas foram semelhantes nos três modelos. Diferenças observáveis, embora pequenas, ocorreram principalmente entre as estimativas obtidas por regressão aleatória e análises bicaracterísticas. As tendências de mudança das variâncias com a idade do animal, como esperado, confirmam aquelas descritas por Albuquerque \& Meyer (2001a,b).

As estimativas de herdabilidade para o efeito direto para os três modelos foram muito próximas para os pesos ao nascimento, aos 120 dias e à desmama (Tabela 2). As maiores diferenças foram observadas nas herdabilidades estimadas para P365 e P550, obtidas empregando-se o modelo que considerou polinômios de Legendre para os quatro efeitos aleatórios (PL). Em geral, esses valores estão de acordo com os apresentados por Mercadante et al. (1995) para a raça Nelore no Brasil. Esses autores descreveram médias ponderadas para herdabilidade do efeito direto de 0,$33 ; 0,22 ; 0,20$ e 0,38 para os pesos ao nascer, à desmama e aos 12 e 18 meses de idade, respectivamente. Lôbo et al. (2000) relataram médias de herdabilidade direta de 0,31 ; 0,$29 ; 0,37$ e 0,35 , respectivamente, para estas características, para raças Zebu e nativas ou naturalizadas em países tropicais.

As estimativas de correlação genética entre os pesos nas diferentes idades tenderam a ser mais próximas quando obtidas com os modelos BC e FP (Tabela 3). As estimativas foram maiores entre os pesos tomados em idades mais próximas e diminuíram quando o intervalo de tempo entre eles aumentou. Entre P365 e P550, as correlações foram próximas à unidade para os três modelos. Independentemente do modelo, as correlações entre PN e pesos pósdesmame foram de moderadas a baixas e indicam que a seleção para pesos nestas idades deve aumentar o PN mais lentamente que seleção para pesos pré-desmama.

As médias dos valores genéticos preditos para os modelos BC foram maiores que para os modelos de regressão aleatória (PLeFP), exceto para P120 (Tabela 4). Em geral, os coeficientes de variação para os valores genéticos foram muito elevados para as características e as maiores variações ocorreram para FP, seguidas pelo modelo PL, o que pode ser constatado pela amplitude dos valores genéticos preditos para cada característica, de acordo com os três modelos empregados.

As correlações de posto entre os valores genéticos (VG) das características estudadas, PN, P120, P205, P365 e $\mathrm{P} 550$, dentro do mesmo modelo variaram de 0,18 a 0,84 para o modelo BC, de 0,02 a 0,98 para o modelo PL e de 0,04 a 0,99 para o modelo FP (Tabela 5). Para o modelo BC, as correlações de posto foram menores que para os modelos de regressão aleatória, exceto para as correlações entre PN e os 

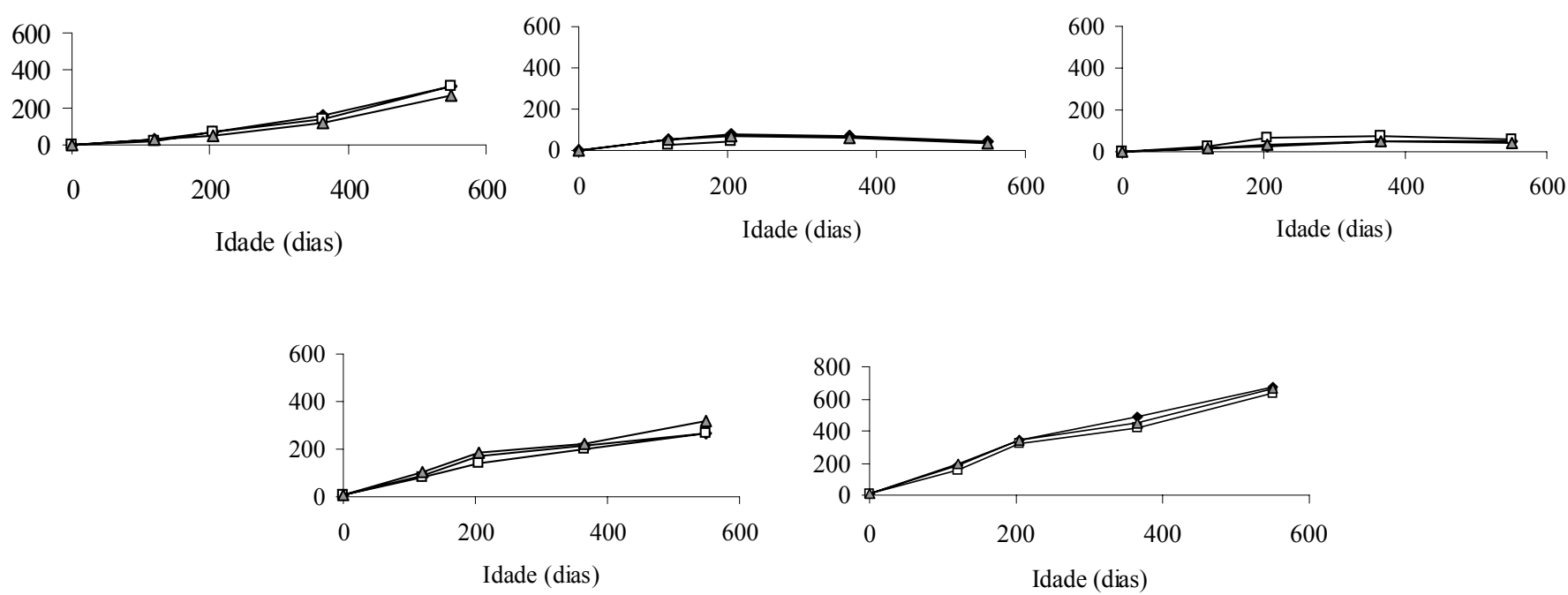

Figura 2 - Estimativas de variância genética direta (acima, esquerda), genética materna (acima, centro), de ambiente permanente materno (acima, direita), de ambiente (abaixo, esquerda) e fenotípica (abaixo, direita) obtidas utilizando-se análise bicaracterísticas padrão $(\square)$, polinômios de Legendre $(\bullet)$ e função de correlação paramétrica $(\boldsymbol{\Delta})$ para modelar o efeito de ambiente permanente de animal.

Tabela 2 - Estimativas de herdabilidade para peso ao nascer (PN) e pesos aos 120 (P120), 205 (P205), 365 (P365) e 550 (P550) dias de idade obtidas por análises bicaracterísticas $(\mathrm{BC})$ com polinômio de Legendre $(\mathrm{PL})$ e função de correlação paramétrica (FP) para o ambiente permanente de animal

\begin{tabular}{lccc}
\hline & \multicolumn{3}{c}{ Herdabilidade } \\
\cline { 2 - 4 } Característica & BC & PL & FP \\
\hline PN & 0,33 & 0,32 & 0,31 \\
P120 & 0,15 & 0,15 & 0,18 \\
P205 & 0,20 & 0,15 & 0,19 \\
P365 & 0,33 & 0,26 & 0,35 \\
P5 50 & 0,49 & 0,40 & 0,47 \\
\hline
\end{tabular}

Tabela 3 - Correlações genéticas diretas entre os pesos ao nascer (PN), aos 120 (P120), 205 (P205), 365 (P365) e 550 (P550) dias de idade estimadas utilizando-se análises bicaracterísticas (BC), polinômio de Legendre $(\mathrm{PL})$ e função de correlação paramétrica $(\mathrm{FP})$ para o ambiente permanente de animal

\begin{tabular}{lccc}
\hline Característica & BC & PL & FP \\
\hline PN $\times$ P120 & 0,77 & 0,73 & 0,78 \\
PN $\times$ P205 & 0,45 & 0,68 & 0,51 \\
PN $\times$ P365 & 0,35 & 0,52 & 0,40 \\
PN $\times$ P550 & 0,36 & 0,44 & 0,36 \\
P120 $\times$ P205 & 0,87 & 0,96 & 0,97 \\
P120 $\times$ P365 & 0,80 & 0,71 & 0,81 \\
P120 $\times$ P550 & 0,68 & 0,55 & 0,69 \\
P205 $\times$ P365 & 0,95 & 0,87 & 0,92 \\
P205 $\times$ P550 & 0,86 & 0,75 & 0,84 \\
P365 $\times$ P550 & 0,99 & 0,98 & 0,98 \\
\hline
\end{tabular}

pesos nas demais idades. Com todos os modelos, principalmente os de regressão aleatória, as correlações entre o $\mathrm{PN}$ e os demais pesos foram baixas e indicam grandes mudanças de classificação dos animais. Para os demais pesos, as correlações de posto foram maiores com os modelos de regressão aleatória e diminuíram com o aumento da distância entre as medidas. Esse resultado era previsível, uma vez que, com estes modelos, impõe-se uma estrutura à matriz de co-variâncias. No caso de análises multicaracterísticas padrão, a matriz de co-variâncias é não estruturada. Embora as correlações genéticas estimadas com o modelo bicaracteríscas tenham obedecido esta estrutura, o mesmo não ocorreu com as correlações de posto. Pelos dois modelos de regressão, as correlações de posto entre as características foram semelhantes.

As correlações de posto entre os valores genéticos obtidos por BC, PL ou FP, considerando todos os animais, foram de médias a altas (Tabela 6). As diferenças nas estimativas de herdabilidades e correlações genéticas entre os três modelos, em geral, foram maiores entre BC e PL que entre BC e FP. Contudo, essas diferenças não se repetiram nas correlações de posto, uma vez que as correlações entre $\mathrm{BC}$ e $\mathrm{PL}$ foram iguais ou superiores às de $\mathrm{BC}$ com $\mathrm{FP}$, provavelmente em virtude de os PL serem mais flexíveis que as FP.

Os valores obtidos para as correlações entre os valores genéticos provenientes da FP e PL foram de 0,92 a 1,00 para touros e para todos os animais. Essas correlações sugerem 
Tabela 4 - Valores genéticos estimados para peso de bovinos Nelore em diferentes idades aplicando-se modelos bicaracterísticos, polinômios de Legendre e funções de correlação paramétricas

\begin{tabular}{|c|c|c|c|c|c|c|}
\hline Característica & Média & DP $(\mathrm{kg})$ & Mediana (kg) & Mínimo & Máximo & Amplitude \\
\hline \multicolumn{7}{|c|}{ Modelo bicaracterístico } \\
\hline $\mathrm{PN}$ & 0,2596 & 1,1890 & 0,1688 & $-4,2632$ & 5,5666 & 9,8298 \\
\hline P 120 & 0,2221 & 2,3982 & 0,0993 & $-8,9207$ & 9,8923 & 18,813 \\
\hline P 205 & 0,6715 & 3,5407 & 0,4191 & $-9,8632$ & 17,2800 & 27,1432 \\
\hline P 365 & 2,3024 & 5,7964 & 1,4313 & $-9,9937$ & 27,3536 & 37,3473 \\
\hline P 550 & 2,7703 & 9,8332 & 2,4465 & $-54,7968$ & 45,7761 & 100,5729 \\
\hline \multicolumn{7}{|c|}{ Modelo de regressão aleatória - Polinômio de Legendre } \\
\hline $\mathrm{PN}$ & 0,1398 & 1,7979 & 0,1082 & $-7,3597$ & 7,7213 & 15,081 \\
\hline P 120 & 0,2845 & 2,5100 & 0,1738 & $-9,8178$ & 11,0257 & 20,8435 \\
\hline $\mathrm{P} 205$ & 0,5320 & 3,4792 & 0,3391 & $-9,9835$ & 16,0898 & 26,0733 \\
\hline P 365 & 0,7517 & 5,8338 & 0,5720 & $-32,5481$ & 28,352 & 60,9001 \\
\hline P 550 & 1,3128 & 9,0393 & 1,0555 & $-54,0019$ & 45,0768 & 99,0787 \\
\hline \multicolumn{7}{|c|}{ Modelo de regressão aleatória - Função de correlação paramétrica } \\
\hline $\mathrm{PN}$ & 0,1362 & 1,6439 & 0,0953 & $-6,6341$ & 7,0490 & 13,6831 \\
\hline P 120 & 0,2695 & 2,8036 & 0,1720 & $-9,5653$ & 13,0168 & 22,5821 \\
\hline P20 5 & 0,6349 & 4,0980 & 0,3339 & $-9,8538$ & 21,4722 & 31,326 \\
\hline P 365 & 0,5734 & 6,8144 & 0,4523 & $-38,9516$ & 30,7050 & 69,6566 \\
\hline P 550 & 1,0508 & 9,9589 & 0,8831 & $-59,7621$ & 47,0305 & 106,7926 \\
\hline
\end{tabular}

Tabela 5 - Correlações de posto (Spearman) entre os valores genéticos dos pesos em diferentes idades obtidas utilizando-se análises bi-características $(B C)$, polinômio de Legendre ( $P L)$ e função de correlação paramétrica (FP) para o ambiente permanente de animal, considerando todos os animais

\begin{tabular}{|c|c|c|c|c|c|c|c|c|c|c|c|c|c|c|c|}
\hline & \multicolumn{5}{|c|}{$\mathrm{BC}$} & \multicolumn{5}{|c|}{ PL } & \multicolumn{5}{|c|}{ FP } \\
\hline & $\mathrm{PN}$ & P 120 & P20 20 & P 365 & P 550 & $\mathrm{PN}$ & P 120 & P20 5 & P365 & P 550 & $\mathrm{PN}$ & P 120 & P2 05 & P365 & P 550 \\
\hline $\mathrm{PN}$ & 1,0 & 0,84 & 0,61 & 0,18 & 0,27 & 1,0 & 0,64 & 0,40 & 0,17 & 0,02 & 1,0 & 0,50 & 0,28 & 0,14 & 0,04 \\
\hline P20 20 & & & 1,0 & 0,61 & 0,58 & & & 1,0 & 0,92 & 0,84 & & & 1,0 & 0,90 & 0,85 \\
\hline P 365 & & & & 1,0 & 0,72 & & & & 1,0 & 0,98 & & & & 1,0 & 0,99 \\
\hline P 550 & & & & & 1,0 & & & & & 1,0 & & & & & 1,0 \\
\hline
\end{tabular}

que a utilização de qualquer desses modelos levaria à seleção de praticamente os mesmos animais. Isso aconteceu mesmo com diferenças de até 0,09 nas estimativas de herdabilidades (P365) e de até 0,17 nas correlações genéticas $(\mathrm{PN} \times \mathrm{P} 205)$ para esses dois modelos (Tabelas 2 e 3$)$. Essas diferenças nos parâmetros estimados se refletem em diferenças nos animais extremos.

As maiores correlações de posto foram observadas para PN e P550. A maior correlação observada para o PN provavelmente se deve ao fato de que este peso não precisa passar por ajustes para idade do animal. Nobre et al. (2003), em pesquisa com um grande conjunto de dados de animais Nelore, encontraram correlações entre os valores genéticos das análises multicaracterísticas e das regressões aleatórias variando de 0,80 a 0,89 para todos os animais. Os resultados encontrados neste trabalho foram semelhantes para PN e P550, mas inferiores aos obtidos por esses autores para os demais pesos. Essa diferença pode estar relacionada ao fato de esses autores terem estimado os valores genéticos analisando todas as características ao mesmo tempo, diferentemente deste trabalho. Nobre et al. (2003) consideraram que estas correlações foram baixas e atribuíram os resultados às diferenças em parâmetros genéticos obtidos pelos dois modelos. Quando os autores estimaram as correlações de posto entre os valores genéticos para touros com progênies, estas correlações foram maiores e variaram de 0,92 a 0,97 .

Comparando ambos os modelos de regressão aleatória (FP e PL) ao modelo padrão (BC) comumente aplicado nas avaliações de bovinos de corte, as correlações de posto foram menores e comprovaram que existem divergências nas classificações dos animais entre esses modelos. Essas divergências eram esperadas e podem ser explicadas pela diferença na abordagem de cada modelo. No caso das análises 
bicaracterísticas, embora elas considerem as correlações entre as características, as matrizes de co-variâncias são nãoestruturadas, diferentemente da abordagem dada pela regressão aleatória. Maior proximidade entre os resultados poderia ser esperada caso as análises fossem realizadas para todas as características conjuntamente, o que tem sido menos utilizado, considerando o elevado número de características, a exigência computacional e a dificuldade de se atingir a convergência. Além disso, com os modelos multicaracterísticos padrão, os pesos devem ser ajustados para idade do animal, enquanto com MRA isso não é necessário, uma vez que a idade está incluída na matriz de delineamentos.

Com base nos valores genéticos preditos pelos modelos PL e FP, observou-se que, selecionando os $10 \%$ melhores machos, 78 (132) a 94\% (158) seriam coincidentes (Tabela 7). Essas porcentagens seriam menores (41 a 94\%) se apenas $1 \%$ dos animais fosse selecionado. Considerando a seleção de $10 \%$ dos touros (animais com progênie), no mínimo $81 \%$ dos animais seriam coincidentes para todas as características. Resultados semelhantes seriam obtidos se apenas 4\% dos touros fossem selecionados. Maiores coincidências seriam esperadas no caso dos touros, uma vez que, por possuírem progênie, esses animais têm valores genéticos preditos com maior acurácia. Comparando os modelos de regressão aleatória (PL e FP) ao modelo padrão (BC), as porcentagens de animais coincidentes seriam menores. Estes achados comprovam que, mesmo com correlações bastante altas entre os valores genéticos, haveria mudanças de classificação, de importância prática ou não. Estudos de simulação poderiam ajudar a quantificar as mudanças que poderiam ser esperadas, em termos de acurácia e resposta à seleção, pelo uso de FP em lugar de PL.

$\mathrm{Na}$ literatura existem diversos trabalhos utilizando modelos de regressão aleatória para estimação de componentes de co-variâncias, mas sua aplicação na avaliação genética dos animais ainda é restrita e, em bovinos, têm sido empregados apenas para gado de leite (Jamrozik et al., 2002; Lidauer et al., 2006). Espera-se que os MRA produzam estimativas de parâmetros genéticos e predições de valores genéticos mais acuradas que os modelos multicaracterísticos utilizados atualmente e que, no futuro, possam substituí-los. Meyer (2004), trabalhando com simulação, verificou que os modelos de regressão aleatória apresentaram maior acurácia (de até $8,3 \%$ ), medida pela correlação dos valores estimados ao simulado, em comparação a um modelo multicaracterístico. $\mathrm{O}$ autor atribuiu esta vantagem à melhor modelagem das variâncias e dos parâmetros genéticos fornecida pelos MRA.

Um aspecto importante a ser considerado em avaliações genéticas de um grande número de animais, como programas de avaliação nacionais, são os requerimentos computacionais. Empregando-se MRA, em vez de modelos multicaracterísticos, haverá aumento no número de equações de modelos mistos, a matriz de coeficientes dos efeitos aleatórios será mais densa e, conseqüentemente, os requerimentos computacionais irão aumentar (Albuquerque \& Meyer, 2001a). Em trabalhos com gado de corte, a modelagem das mudanças das variâncias de ambiente permanente com a idade tem exigido o emprego de polinômios de ordem mais alta que para os demais efeitos (Meyer, 2001a; Albuquerque \& Meyer, 2001a). Os resultados deste trabalho indicam que um modelo de correlação paramétrico não estacionário é uma alternativa para modelar a variância dentro de animal e diminuir o número de parâmetros necessários para descrever as mudanças nas co-variâncias com a idade (Meyer, 2001b).

\section{Conclusões}

As correlações de posto usando os diferentes métodos indicam que ocorrem diferenças de classificação dos animais quando os modelos de regressão aleatória são compa-

Tabela 6 - Correlações de posto (Spearman) entre os valores genéticos preditos utilizando-se análises bicaracterísticas (BC), polinômio de Legendre (PL) e função de correlação paramétrica (FP) para o ambiente permanente de animal, considerando todos os animais e apenas os touros

\begin{tabular}{|c|c|c|c|c|c|c|}
\hline Característica & \multicolumn{3}{|c|}{ Todos os animais (5.751) } & \multicolumn{3}{|c|}{ Touros (260) } \\
\hline $\mathrm{PN}$ & 0,81 & 0,84 & 1,00 & 0,75 & 0,78 & 1,00 \\
\hline P 205 & 0,73 & 0,65 & 0,92 & 0,75 & 0,72 & 0,98 \\
\hline P 365 & 0,64 & 0,65 & 0,98 & 0,59 & 0,58 & 0,99 \\
\hline P 550 & 0,87 & 0,89 & 0,98 & 0,87 & 0,88 & 0,99 \\
\hline
\end{tabular}


Tabela 7 - Número de machos e de touros que seriam selecionados utilizando-se os valores genéticos obtidos por análises bicaracterísticas $(B C)$, polinômio de Legendre (PL) e função de correlação paramétrica (FP) para o ambiente permanente de animal aplicandose diferentes intensidades de seleção

\begin{tabular}{lcccc}
\hline Característica & \multicolumn{2}{c}{$\%$ de machos selecionados } & \multicolumn{2}{c}{ de touros selecionados } \\
\cline { 2 - 3 } & $10 \%(169$ animais $)$ & $1 \%(17$ animais $)$ & $10 \%(26$ touros $)$ & $4 \%(11$ touros $)$ \\
\hline
\end{tabular}

Peso ao nascer

\begin{tabular}{lcccc}
$\mathrm{BC}-\mathrm{FP}$ & $(104) 61 \%$ & $(5) 29 \%$ & $(17) 65 \%$ & $(8) 73 \%$ \\
$\mathrm{BC}-\mathrm{PL}$ & $(98) 58 \%$ & $(6) 35 \%$ & $(16) 62 \%$ & $(8) 73 \%$ \\
$\mathrm{PL}-\mathrm{FP}$ & $(158) 94 \%$ & $(16) 94 \%$ & $(25) 96 \%$ & $(10)(91 \%)$ \\
\hline
\end{tabular}

Peso aos 120 dias de idade

\begin{tabular}{lccll}
$\mathrm{BC}-\mathrm{FP}$ & $(90) 53 \%$ & $(1) 6 \%$ & $(15) 58 \%$ & \\
$\mathrm{BC}-\mathrm{PL}$ & $(117) 69 \%$ & $(8) 47 \%$ & $(18) 69 \%$ & $(6) 55 \%$ \\
$\mathrm{PL}-\mathrm{FP}$ & $(132) 78 \%$ & $(7) 41 \%$ & $(21) 81 \%$ & $(7) 64 \%$ \\
\hline
\end{tabular}

Peso aos 205 dias de idade

\begin{tabular}{lcccc}
$\mathrm{BC}-\mathrm{FP}$ & $(81) 47 \%$ & $(6) 35 \%$ & $(14) 54 \%$ & $(8) 73 \%$ \\
$\mathrm{BC}-\mathrm{PL}$ & $(92) 54 \%$ & $(5) 29 \%$ & $(17) 65 \%$ & $(8) 73 \%$ \\
$\mathrm{PL}-\mathrm{FP}$ & $(136) 81 \%$ & $(13) 76 \%$ & $(22) 85 \%$ & $(11) 100 \%$ \\
\hline
\end{tabular}

Peso aos 365 dias de idade

\begin{tabular}{lcccc}
$\mathrm{BC}-\mathrm{FP}$ & $(80) 47 \%$ & $(4) 24 \%$ & $(8) 31 \%$ & $(2) 18 \%$ \\
$\mathrm{BC}-\mathrm{PL}$ & $(78) 46 \%$ & $(4) 24 \%$ & $(9) 35 \%$ & $(2) 18 \%$ \\
$\mathrm{PL}-\mathrm{FP}$ & $(143) 85 \%$ & $(12) 71 \%$ & $(21) 81 \%$ & $(10) 91 \%$ \\
\hline
\end{tabular}

Peso aos 550 dias de idade

\begin{tabular}{lllll}
$\mathrm{BC}-\mathrm{FP}$ & $(125) 74 \%$ & $(11) 65 \%$ & $(17) 65 \%$ & $(7) 64 \%$ \\
$\mathrm{BC}-\mathrm{PL}$ & $(121) 72 \%$ & $(10) 59 \%$ & $(17) 65 \%$ & $(8) 73 \%$ \\
$\mathrm{PL}-\mathrm{FP}$ & $(149) 88 \%$ & $(14) 82 \%$ & $(25) 96 \%$ & $(10) 91 \%$ \\
\hline
\end{tabular}

rados ao método usual empregado em avaliações genéticas. Funções de correlação paramétricas são uma alternativa viável aos polinômios de Legendre para modelar os efeitos de ambiente permanente de animal e permitem diminuição no número de parâmetros a serem estimados.

\section{Literatura Citada}

ALBUQUERQUE, L.G.; MEYER, K. Estimates of covariance functions for growth from birth to 630 days of age in Nelore cattle Journal of Animal Science, v.79, p.2776-2789, 2001a.

ALBUQUERQUE, L.G.; MEYER, K. Estimates of direct and maternal genetic effects for weights from birth to 600 days of age in Nelore cattle. Journal of Animal Breeding and Genetics, v.118, p.83-92, 2001b.

ALBUQUERQUE, L.G.; MEYER, K. Estimates of covariance functions for growth of Nelore cattle applying a parametric correlation function to model within-animal correlations. Livestock Production Science, v.93, p.213-222, 2005.

BOLDMAN, K.G.; KRIESE, L.A., Van VLECK, L.D. et al. A manual for use of MTDFREML. Clay Center: USDA - ARS, 1993. 120p.

DIAS, L.T.; ALBUQUERQUE, L.G.; TONHATI, H. et al. Estimação de parâmetros genéticos para peso do nascimento aos 550 dias de idade para animais da raça Tabapuã utilizando modelos de regressão aleatória. Revista Brasileira de Zootecnia, v.35, n.5, p.1915-1925, 2006.

FOULLEY, J.L.; JAFFRÉZIC, F.; ROBERT-GRANIÉ, C. EM-REML estimation of covariance parameters in Gaussian mixed models for longitudinal data analysis. Genetic Selection Evolution, v.32, p.129-141, 2000 .
GRASER, H.U.; SMITH, S.P.; TIER, B. A derivative-free approach for estimating variance components in animal model by restricted maximum likelihood. Journal of Animal Science, v.64, p.1362-1370, 1987.

JAMROZIK, J.; SCHAEFFER, L.R.; DEKKERS, J.C.M. Genetic evaluation of dairy cattle using test day yields and random regression model. Journal of Dairy Science, v.80, p.1217-1226, 1997.

JAMROZIK, J.; SCHAEFFER, L.R.; WEIGEL, K.A. Genetic evaluation of bulls and cows with single- and multiple-country test-day models. Journal of Dairy Science, v.85, p.16171622, 2002.

KIRKPATRICK, M.; HECKMAN, N. A quantitative genetic model for growth, shape and other infinite-dimensional characters. Journal Mathematical Biology, v.27, p.429-450, 1989.

KIRKPATRICK, M.; LOFSVOLD, D.; BULMER, M. Analysis of the inheritance, selection and evolution of growth trajectories. Genetics, v.124, p.979-993, 1990.

KIRKPATRICK, M.; HILL, W.G.; THOMPSON, R. Estimating the covariance structure of traits during growth and aging, illustrated with lactations in dairy cattle. Genetic Research, v.64, p.5769, 1994.

KOOTS, K.R.; GIBSON, J.P.; SMITH, C. et al. Analyses of published genetic parameter estimates for beef production traits. 1. Heritability. Animal Breeding Abstracts, v.62, p.309-338, $1994 \mathrm{a}$.

KOOTS, K.R.; GIBSON, J.P.; SMITH, C. et al. Analyses of published genetic parameter estimates for beef production traits. 2. Phenotypic and genetic correlations. Animal Breeding Abstracts, v.62, p.309-338, 1994b.

LIDAUER, M.; PEDERSEN, J.; PÖSO, J. et al. [2006]. Joint Nordic test day model: evaluation model. Disponível em: $<\mathrm{http}: / /$ www-interbull.slu.se/framesida-home.htm, publications $>$ Acesso em: $31 / 10 / 2006$. 
LÔBO, R.N.B.; MADALENA, F.E.; VIEIRA, A.R. Average estimates of genetic parameters for beef and dairy cattle in tropical regions. Animal Breeding Abstracts, v.68, p.433-462, 2000.

LÓPES-ROMERO, P.; CARABAÑO, M.J. Comparing alternative random regression models to analyze first lactation daily milk yield data in Holstein-Friesian cattle. Livestock Production Science, v.82, p.81-96, 2003.

MERCADANTE, M.E.Z.; LÔBO, R.B.; REYES, A. Parámetros genéticos para características de crecimiento en cebuínos de carne: una revisión. Archivo Latinoamericano Producción Animal, v.3, p.45-89, 1995.

MEYER, K. Estimating covariance functions for longitudinal data using a random regression model. Genetic Selection Evolution, v.30, p.221-240, 1998a.

MEYER, K. "DXMRR"- a program to estimate covariance functions for longitudinal data by restricted maximum likelihood. In: WORLD CONGRESS ON GENETICS APPLIED TO LIVESTOCK PRODUCTION, 6., 1998, Armidale. Proceedings... Armidale, 1998b, v.27. p.465-466.

MEYER, K. Modeling 'repeated' records: covariance functions and random regression models to analyze animal breeding data. In: WORLD CONGRESS ON GENETICS APPLIED TO LIVESTOCK PRODUCTION, 6. 1998, Armidale. Proceedings... Armidale, 1998c. v.25, p.517-520.

MEYER, K. Estimates of genetic and phenotypic covariance functions for postweaning growth and mature weight of beef cows. Journal of Animal Breeding and Genetics, v.116, p.181-205, 1999.

MEYER, K. Random regressions to model phenotypic variation in monthly weights of Australian beef cows. Livestock Production Science, v.65, p.19-38, 2000.

MEYER, K. Estimates of direct and maternal covariance functions for growth of Australian beef calves from birth to weaning. Genetic Selection Evolution, v.33, p.1-28, 2001 a.

MEYER, K. Estimating genetic covariance functions assuming a parametric correlation structure for environmental effects. Genetic Selection Evolution, v.33, p.557-585, 2001 b.

MEYER, K. Scope for a random regression model in genetic evaluation of beef cattle for growth. Livestock Production Science, v.86, p.69-83, 2004

MEYER, K.; HILL, W.G. Estimation of genetic and phenotypic covariance functions for longitudinal or 'repeated' records by restricted maximum likelihood. Livestock Production Science, v.47, p.185-200, 1997.
MOHIUDDIN, G. Estimates of genetic and phenotypic parameters of some performance traits in beef cattle. Animal Breeding Abstracts, v.61, p.495-522, 1993.

NOBRE, P.R.C.; MISZTAL, I.; TSURUTA, S. et al. Genetic evaluation of growth in Nelore cattle by multiple-trait and random regression models. Journal of Animal Science, v. 81 , p.927-932, 2003.

OLORI, V.E.; HILL, W.G.; MCGUIRK, B.J. et al. Estimating variance components for test day milk records by restricted maximum likelihood with random regression animal model. Livestock Production Science, v.61, p.53-63, 1999.

PATTERSON, H.D.; THOMPSON, R. Recovery of inter-block information when block sizes are unequal. Biometrika, v.58, p.545-54, 1971 .

SCARPEL, L.C.P. Estimativas de parâmetros e valores genéticos para peso corporal de bovinos da raça Guzerá usando-se regressão aleatória. Jaboticabal: Universidade Estadual Paulista, 2004. 70p. Tese (Doutorado em Zootecnia) - Universidade Estadual Paulista, 2004.

SAKAGUTI, E.S.; SILVA, M.A.; QUAAS, R.L. et al. Avaliação do crescimento de bovinos jovens da raça Tabapuã, por meio de análises de funções de covariâncias. Revista Brasileira de Zootecnia, v.32, n.4, p.864-874, 2003.

SCHAEFFER, L.R.; DEKKERS, J.C.M. Random regressions in animal models for test-day production in dairy cattle. In WORLD CONGRESS ON GENETICS APPLIED TO LIVESTOCK PRODUCTION, 5., 1994, Guelph, Proceedings... Guelph: 1994. v.17, p.443-446.

SILVA, I.S. Componentes de (co)variância e parâmetros genéticos para características de crescimento de bovinos da raça Guzerá usando diferentes estratégias de análise. Piracicaba: Escola Superior de Agricultura Luis de Queiroz, 2004. 106p. Tese (Doutorado em Ciência Animal e Pastagem) - Escola Superior de Agricultura Luis de Queiroz, 2004.

SMITH, S.P.; GRASER, H.-U. Estimating variance components in a class of mixed models by restricted maximum likelihood. Journal of Dairy Science, v.69, p.990-995, 1986.

STRABEL, T.; SZYDA, J.; PTAK, E. et al. Comparison of random regression test-day models for Polish Black and White cattle. Journal of Dairy Science, v.88, p.3688-3699, 2005.

Van der WERF, J.H.J.; GODDARD, M.E.; MEYER, K. The use of covariance functions and random regressions for genetic evaluation of milk production based on test day records. Journal of Dairy Science, v.81, p.3300-3308, 1998. 